
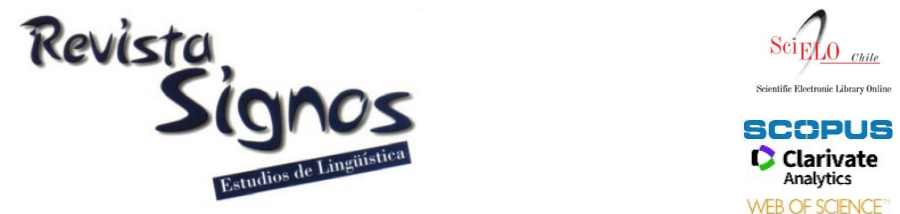

WEB OF SCIENCE

\title{
Los neologismos desde una perspectiva funcional: Correlación entre percepción y datos empíricos $^{1}$
}

\author{
Neologisms from a functional perspective: Correlation between \\ perception and empirical data
}

\author{
Elisabet Llopart-Saumell \\ UNIVERSiTAT D'AlaCANT, ALACANT \\ UNIVERSITAT POMPEU FABRA, BARCELONA \\ ESPAÑA \\ elisabet.llopart@ua.es
}

Recibido: 08-IV-2018 / Aceptado: 05-XII-2018

DOI: $10.4067 /$ S0718-09342019000200665

\section{Resumen}

Este trabajo se centra en la función de los neologismos y, más concretamente, pone en entredicho la aplicabilidad de la dicotomía tradicional 'neologismo denominativo' y 'neologismo estilístico'. Aunque se trata de una clasificación ampliamente citada en la bibliografía sobre neología, tanto desde un punto de vista teórico como práctico presenta ciertas limitaciones que ponen en duda que se trate realmente de categorías opuestas (Llopart-Saumell, 2016). El contenido de este estudio parte de una encuesta lingüística basada en la percepción de los hablantes: se pide a los participantes que clasifiquen un conjunto de neologismos en catalán (en contexto) en denominativos, estilísticos o con ambos valores (denominativos y estilísticos). A continuación, se miden los grados de coincidencia y, finalmente, los resultados se contrastan con datos sociolingüísticos (frecuencia y estabilidad en el uso), discursivos (contexto y posición discursiva) y lingüísticos (morfosemánticos) de cada uno de los neologismos del estudio. Los resultados obtenidos ponen de relieve que los neologismos considerados estilísticos presentan un grado de coincidencia más alto que los considerados denominativos, puesto que presentan determinados rasgos, especialmente lingüísticos y discursivos, que parece que los hace más fácilmente identificables. En cambio, en la clasificación de los neologismos como denominativos hay menos coincidencia y aumenta el número de unidades clasificadas con ambas funciones, denominativa y estilística. En cuanto a los diferentes aspectos estudiados en correlación con los resultados, se observa que los diferentes criterios no funcionan de forma autónoma, sino que se complementan entre sí. 
Palabras Clave: Neología, neologismo denominativo, neologismo estilístico, intuición lingüística, función de los neologismos.

\begin{abstract}
This research focuses on the function of neologisms and casts serious doubts on the applicability of the traditional dichotomy 'denominative neologisms' and 'stylistic neologisms'. Although this classification is widely mentioned in the literature on neology, both from a theoretical and applied perspective it shows some limitations that call into question that they really work as opposing categories (Llopart-Saumell, 2016). The starting point of this study is a linguistic survey based on the perception of the speakers: participants were asked to classify a set of neologisms in Catalan (in context) in denominative, stylistic or with both values (denominative and stylistic). Then, we study the degree of coincidence of the results obtained and, finally, we contrast them with sociolinguistic (frequency and stability of use), discursive (context and discursive position) and linguistic data (morpho-semantic) of each of the neologisms. The results point out that those neologisms considered stylistic have a higher degree of coincidence than those considered denominative, because they share some features, especially linguistic and discursive, that seem to make them easier to be identified as such. In this sense, those neologisms classified as denominative show a lower degree of coincidence and the number of units classified with both values increases. Regarding the different aspects contrasted with the results, we can observe that the different criteria do no not work autonomously, but they complement each other.
\end{abstract}

Key Words: Neology, denominative neologism, stylistic neologism, linguistic intuition, function of neologisms.

\title{
INTRODUCCIÓN
}

En el estudio de la neología como campo de conocimiento que se ocupa de las palabras nuevas, es decir, creadas en un período determinado en una lengua concreta, los conceptos más utilizados para diferenciar unos tipos de neologismos de otros de acuerdo con su función comunicativa son los de 'neología denominativa' y 'neología estilística'. Varios autores, como Guilbert (1975), Cabré (1989), Sablayrolles (1993) y Guerrero Ramos (1995), han hecho referencia a estos dos conceptos y en la mayoría de los casos los han relacionado entre sí en forma de categorías explícitamente opuestas, es decir, como una dicotomía de la función neológica. Ahora bien, aunque estos dos conceptos han sido ampliamente utilizados en la literatura, podemos afirmar que, desde un punto de vista teórico, la clasificación de los neologismos en denominativos y estilísticos no se sustenta: las funciones de los neologismos no se inscriben en un único eje en que los neologismos prototípicamente referenciales se inscriben en un extremo y los prototípicos de la función estilística en el otro, y tampoco podemos separar en dos ejes los neologismos denominativos y los estilísticos. En este sentido, parece que todos los neologismos están motivados referencialmente, pero algunos también llevan a cabo otras funciones (LlopartSaumell, 2016). 
Aunque parece que esta dicotomía responde a cierta intuición lingüística, a la práctica no siempre permite obtener resultados satisfactorios, porque aparecen dudas de clasificación de acuerdo con las características lingüísticas y extralingüísticas que pueden considerarse denominativas o estilísticas. En este sentido, la dificultad para definir de forma teórica al neologismo o innovación léxica tiene su correlato también en la práctica. En relación con la función de los neologismos, ocurre lo mismo, las limitaciones teóricas para explicar de forma teórica este fenómeno también se observan de forma aplicada. En algunas obras, los autores clasifican los neologismos en denominativos o estilísticos, pero no queda claro en qué criterios se basan y, además, pueden no coincidir con la percepción del lector. En relación con la intuición lingüística, los estudios de percepción son relevantes para obtener datos sobre las tareas de procesamiento y clasificación del léxico. En las encuestas realizadas para identificar los neologismos (Gardin, Lefèvre, Marcellesi \& Mortureux, 1974; Sablayrolles, 2003; Llopart-Saumell, Loureda, Cabré \& Freixa, 2014; Bernal, 2015) como también en un estudio para identificar los neologismos con rarezas o extraños (Freixa, 2010) se observa que los resultados de percepción de los hablantes no siempre son coincidentes. Por ejemplo, en Bernal (2015) no hay ningún caso de coincidencia absoluta entre los 30 participantes y los casos con coincidencia alta (entre 28 y 27 informantes) representan un 9,09\%, con 4 neologismos. En Freixa (2010), el grado de coincidencia absoluta es del 48\% (con 10 participantes): 42 casos con respuesta negativa y 6, positiva. En Llopart-Saumell et al. (2014) la coincidencia sube, pero es del $50,6 \%$ (12 participantes).

El objetivo principal de este trabajo consiste en demostrar que, de acuerdo con las descripciones que se han llevado a cabo en relación con la función comunicativa de los neologismos, estas unidades no se pueden clasificar de forma sistemática en 'neologismos denominativos', por un lado, y 'neologismos estilísticos', por el otro. A partir de esta premisa, elaboramos una encuesta para estudiar la percepción de los hablantes a la hora de clasificar los neologismos en denominativos y estilísticos. Además, como consideramos que no se trata de una dicotomía, también incluimos una tercera categoría para indicar que los neologismos poseen rasgos de ambos tipos. Concretamente, presentamos una serie de neologismos con su contexto de uso a un grupo de estudiantes universitarios del grado de Traducción e Interpretación, y de Lenguas Aplicadas. Después de haber descrito esta clasificación en clase de acuerdo con las características mencionadas por Guilbert (1975) y Cabré (1989) (que se indican en la Tabla 1), les pedimos que etiqueten las unidades de acuerdo con la función que creen que llevan a cabo en el contexto en que se han producido. Estos resultados permiten, en primer lugar, observar cómo los participantes han clasificado los neologismos y, en segundo lugar, estudiar el grado de coincidencia. Además, posteriormente, correlacionamos los resultados de la encuesta con otros datos que en la bibliografía se utilizan para describir y diferenciar los 'neologismos denominativos', por un lado, y los 'neologismos estilísticos', por el otro. Se trata de aspectos 
sociolingüísticos, como la frecuencia y estabilidad en el uso, observables a partir de corpus textuales y bases de datos, entre otros recursos; aspectos discursivos, como la posición discursiva y las marcas de uso del contexto discursivo, que nos da información sobre la intención comunicativa a partir de rasgos lingüísticos observables en el contexto de uso; y, aspectos lingüísticos, como el tipo de formación y los rasgos morfosemánticos y pragmáticos.

\section{Marco teórico: La función de los neologismos}

\subsection{Clasificación}

De acuerdo con las aportaciones de los autores que han descrito estas dos categorías más ampliamente (Guilbert, 1975; Cabré, 1989; Guerrero Ramos, 1995), en líneas generales, se puede considerar que un neologismo es denominativo si designa un concepto nuevo, propio de la realidad que nos rodea y que tenemos la necesidad de denominar, mientras que los neologismos que expresan una visión subjetiva de una entidad ya conocida se pueden calificar de estilísticos. Ahora bien, las descripciones que se han llevado a cabo hasta ahora no siempre permiten diferenciar un neologismo denominativo de uno de estilístico.

En este sentido, esta clasificación ha recibido diferentes críticas, y una de las principales niega que se trate de conceptos dicotómicos (Faura, 1998; Isla, 2006; Estornell, 2009). Además, otros autores han utilizado funciones y perspectivas diferentes para explicar la función comunicativa de los neologismos (Mounin, 1990; Sablayrolles, 1993, 2000). Algunas de las funciones mencionadas son la economía lingüística y el prestigio o esnobismo (Mounin, 1990; Veny, 1991; Sablayrolles, 1993; García Platero, 1996; Freixa, 2002). Finalmente, Mounin (1990) también indica el uso lúdico del lenguaje, mencionado también por otros autores (Guilbert, 1975; Cabré, 1989; Sablayrolles, 1993; Mejri, 1995). Por su parte, Sablayrolles (1993, 2000) hace referencia a un conjunto de funciones de los neologismos más concretas, por ejemplo, para inculcar una idea o provocar una emoción determinada en el receptor y, en relación con la lengua, señala la precisión, es decir, el hecho de hacer referencia a un concepto más específico, entre otras motivaciones. Como veremos, algunas de estas motivaciones, como la economía lingüística, no se alinean con una de estas dos funciones. Además, hay palabras que no se asocian a ninguno de los dos tipos de neologismos, como los llamados neologismos gramaticales o de lengua, de acuerdo con Guilbert (1975). En este sentido, el autor hace referencia a las palabras que, aunque son nuevas, solo presentan cambios gramaticales (conversión, cambio sintáctico y algún tipo de afijación altamente predecible y, por tanto, imperceptible) y lo ejemplifica con un caso citado por Saussure: indécorable, que se define por las reglas morfosintácticas y pasa desapercibido como neologismo. Cañete (2015), por ejemplo, aunque parte de la dicotomía 'neologismo denominativo' y 'neologismo estilístico' propone la 'función cero', que incluye este grupo de unidades. 
En síntesis, a partir de la revisión aportada hasta ahora podemos ver que las críticas que recibe la concepción de estas categorías en forma de dicotomía se centran en la difícil aplicación práctica de la distinción entre 'neologismos denominativos' y 'neologismos estilísticos', y 'neologismos de lengua', que se trata de un criterio que no se basa en términos funcionales, sino lingüísticos (Mortureux, 1984). En esta línea, algunos autores señalan la arbitrariedad de la oposición de los dos conceptos (Faura, 1998; Isla, 2006), mientras que otros destacan específicamente la dificultad de trazar una frontera entre estas categorías de acuerdo con sus características: si el neologismo es necesario por motivos objetivos o de denominación (en relación con la neología denominativa) o necesario por motivos subjetivos o expresivos (relacionado con la neología estilística) (Casasús, 1992; García Platero, 2016)

\subsection{Parámetros de análisis}

Para demostrar las limitaciones que ofrece esta propuesta de clasificación, LlopartSaumell (2016) ha profundizado en la revisión de los conceptos utilizados para describir y diferenciar los neologismos denominativos, por un lado, y los estilísticos, por el otro. A continuación, presentamos un esquema de acuerdo con Guilbert (1975) y Cabré (1989):

Tabla 1. Dicotomía neologismo denominativo y neologismo estilístico.

\begin{tabular}{|l|l|}
\hline \multicolumn{1}{|c|}{ Neología denominativa } & \multicolumn{1}{c|}{ Neología estilística } \\
\hline Función referencial & Función expresiva \\
\hline Comunicar la realidad & Mostrar la visión personal del autor \\
\hline Adecuarse al referente que designa & Llamar la atención del receptor \\
\hline Uso de formas productivas y usuales & Uso de formas no productivas y transgresoras \\
\hline Frecuencia de uso considerable & Acostumbra a ser efímera \\
\hline Responde a una necesidad objetiva & Responde a una necesidad subjetiva \\
\hline
\end{tabular}

Las características utilizadas se centran en las funciones del lenguaje de acuerdo con Jakobson. A partir de cada una de estas funciones, relacionadas con las nociones de denominación y denotación (la función referencial) y connotación (la función expresiva y la poética) se han aislado una serie de aspectos gramaticales y discursivos. En cuanto a la bibliografía sobre neología, también se ha relacionado uno y otro tipo de neologismos con aspectos sociolingüísticos, como son la frecuencia y la estabilidad en el uso.

En cuanto a los aspectos discursivos, se ha partido de las bases del análisis del discurso (Van Dijk, 1989; Brown \& Yule, 1991; Cortés \& Camacho, 2003) y de la pragmática (Escandell-Vidal, 1996, 2014; Calsamiglia \& Tusón, 2012) para estudiar la función de los neologismos en su contexto de uso, es decir, en que el neologismo no se analiza de forma aislada, sino a partir de un enunciado concreto fruto de una situación comunicativa determinada. En esta línea, se ha revisado el tipo de texto y los géneros de la prensa del ámbito periodístico para conocer cuál es la finalidad de cada 
uno de los géneros y también de las diferentes partes de la noticia. De este modo, hemos podido comprobar que cada uno de estos textos tiene un objetivo diferenciado que, en líneas generales, se puede relacionar con un mayor o menor grado de neologismos y con un valor denominativo o estilístico. Lo mismo sucede en relación con los títulos y el cuerpo de la noticia; cada uno de estos fragmentos tiene una finalidad diferente.

Finalmente, en cuanto a los aspectos gramaticales, de acuerdo con el objetivo general de los diarios, que consiste en informar, algunos rasgos lingüísticos son esperables, en relación con la neutralidad, la objetividad y el orden canónico, mientras que otros no son representativos de este tipo de texto, como la expresividad, la informalidad, la subjetividad y la deixis de emisor. Por lo tanto, si en un fragmento del texto se registran elementos de este segundo tipo nos indica que el texto tiene una intención que no consiste solo en informar, sino también en persuadir, convencer, conmover, etc. Se trata, pues, de aspectos relacionados con el registro, el estilo y la subjetividad. En último lugar, en relación con los aspectos lingüísticos o morfológicos, los neologismos denominativos suelen presentar formas productivas mientras que los estilísticos, como quieren llamar la atención, priorizan las formas originales y poco productivas. En este sentido, pueden desviarse de las normas establecidas. Por este motivo, estudiamos el concepto de productividad y norma.

\section{Método del estudio}

\subsection{Participantes}

La encuesta fue realizada por 25 estudiantes $^{2}$ de la asignatura de Léxico del cuarto curso del grado de Traducción e Interpretación, y de Lenguas Aplicadas, durante el año académico 2014-2015 en la Universidad Pompeu Fabra de Barcelona.

Tabla 2. Sexo de los participantes.

\begin{tabular}{|l|c|c|}
\hline \multicolumn{1}{|c|}{ Sexo } & Total & $\mathbf{\%}$ \\
\hline mujer & 20 & 83,3 \\
\hline hombre & 4 & 16,7 \\
\hline
\end{tabular}

Entre los participantes destacan las mujeres, ya que representan más de tres cuartas partes del total. Este hecho se explica porque la mayoría de los estudiantes de la Facultad de Traducción e Interpretación son mujeres.

Tabla 3. Lengua inicial de los participantes.

\begin{tabular}{|l|c|c|}
\hline \multicolumn{1}{|c|}{ Lengua inicial } & Total & $\mathbf{\%}$ \\
\hline catalán & 13 & 54,2 \\
\hline castellano & 8 & 33,3 \\
\hline catalán y castellano & 2 & 8,3 \\
\hline otras & 2 & 8,3 \\
\hline
\end{tabular}


Si nos fijamos en la lengua materna de los estudiantes, un poco más de la mitad declara que su lengua inicial es el catalán mientras que el castellano representa una tercera parte del total. Algunos participantes indican que poseen ambas lenguas como iniciales $(8,3 \%)$ y este mismo porcentaje se observa entre los que declaran que su lengua inicial es otra (en un caso, el inglés y, en otro, el rumano).

Tabla 4. Edad de los participantes.

\begin{tabular}{|l|c|c|}
\hline \multicolumn{1}{|c|}{ Edad } & Total & $\mathbf{\%}$ \\
\hline 21 & 17 & 70,8 \\
\hline 22 & 3 & 12,5 \\
\hline 23 & 2 & 8,3 \\
\hline otra & 2 & 8,3 \\
\hline promedio & & 21,9 \\
\hline
\end{tabular}

Finalmente, respecto de la edad, el promedio se sitúa en 21,9 años, que se corresponde con los años que tienen la mayoría de los alumnos en el último curso del grado.

\subsection{Materiales}

El ejercicio que se distribuyó entre los participantes de este estudio está formado por 14 neologismos con su correspondiente contexto de uso. De forma más detallada, los neologismos se obtuvieron del banco de neologismos del Observatori de Neologia (BOBNEO) y proceden de periódicos generales en catalán publicados entre 2010 y 2012. Las unidades se seleccionaron de acuerdo con el criterio lexicográfico, es decir, se trata de palabras no documentadas en los principales diccionarios de referencia en catalán (el DIEC2 y el GDLC) ${ }^{3}$.

Tabla 5. Neologismos de la encuesta.

\begin{tabular}{|l|l|l|}
\hline \multicolumn{1}{|c|}{ Neologismos } & C. g. & \multicolumn{1}{|c|}{ Tipo de formación } \\
\hline autoretallada 'autorecorte' & $\mathrm{f}$ & composición culta \\
\hline caçavots 'cazavotos' & $\mathrm{adj}$ & composición patrimonial \\
\hline catalanofobia 'catalanofobia' & $\mathrm{f}$ & composición culta \\
\hline claqueter claquetera 'claquetero claquetera' & $\mathrm{m} \mathrm{y} \mathrm{f}$ & sufijación \\
\hline cleptòcrata 'cleptócrata' & $\mathrm{m} \mathrm{y} \mathrm{f}$ & composición culta \\
\hline cretinitzador - a 'cretinizador -ra' & $\mathrm{adj}$ & sufijación \\
\hline méteo 'méteo' & $\mathrm{f}$ & abreviación \\
\hline onze de garanties 'once de garantias' & $\mathrm{m}$ & sintagmación \\
\hline pseudoecologista 'pseudoecologista' & $\mathrm{adj}$ & prefijación \\
\hline rastaflauta 'perroflauta' & $\mathrm{m} \mathrm{y} \mathrm{f}$ & composición patrimonial \\
\hline saxofonic - a 'saxofónico -ca' & $\mathrm{adj}$ & sufijación \\
\hline seminacionalitzar 'seminacionalizar' & $\mathrm{v} \mathrm{tr}$ & prefijación \\
\hline sou base 'sueldo base' & $\mathrm{m}$ & composición patrimonial \\
\hline webisodi 'webisodio' & $\mathrm{m}$ & acronimia \\
\hline
\end{tabular}


Como en otros estudios de percepción, los neologismos se han seleccionado de modo que sean representativos de los diferentes tipos de formación. Además, los rasgos lingǘsticos que analizaremos posteriormente (frecuencia de uso, documentación en otras obras, posición y contexto discursivo, y aspectos morfosemánticos) se encuentren representados de forma equilibrada. Desde un punto de vista cuantitativo, la muestra de neologismos seleccionados se asemeja a las de otros cuestionarios lingüísticos que hemos tomado como referencia (Varo, 2013, con 16 unidades; Sánchez Manzanares, 2013, que cuenta con 10 palabras nuevas; y Estopà, 2015, con 20 neologismos). Esta encuesta exploratoria permite establecer correlaciones que, a su vez, son útiles para identificar y aislar variables para posteriores estudios.

\subsection{Procedimiento}

En el marco de la clase de Léxico, se explicó a los estudiantes qué son los neologismos y, a continuación, se detalló la dicotomía usada para clasificar estas unidades de acuerdo con su función comunicativa en 'neologismos denominativos' y 'neologismos estilísticos'. Para ello, se partió de las descripciones de Guilbert (1975) y Cabré (1989), que se han mencionado anteriormente y se resumen en la Tabla 1.

De acuerdo con esta dicotomía ampliamente citada en la bibliografía, se pidió a cada uno de ellos que, de forma individual, clasificara los 14 neologismos, de acuerdo con su contexto de uso, en denominativos y estilísticos. $\mathrm{O}$, si creían que las unidades mostraban características de ambos tipos, podían indicar ambas categorías en la casilla correspondiente. No se señalaron las posibles dificultades que podrían tener para para no interferir en el proceso de decisión léxica, ya que podría sesgar los resultados.

\section{Análisis de los resultados}

\subsection{Clasificación y grado de coincidencia}

A continuación, presentamos los resultados obtenidos a partir de las encuestas realizadas. En este sentido, podemos ver cuántos participantes clasificaron como denominativos, estilísticos o con ambos valores cada uno de los 14 neologismos de la encuesta. Además, indicamos qué porcentaje representa para cada una de las categorías. 
Tabla 6. Resultados cuantitativos de la encuesta.

\begin{tabular}{|c|c|c|c|c|c|c|}
\hline \multirow{2}{*}{ Neologismos } & \multicolumn{2}{|c|}{ Denominativos } & \multicolumn{2}{|c|}{ Estilísticos } & \multicolumn{2}{|c|}{ Ambos } \\
\hline & Total & $\%$ & Total & $\%$ & Total & $\%$ \\
\hline autoretallada 'autorecorte', $\mathrm{f}$ & 15 & 65,2 & 6 & 26,1 & 2 & 8,7 \\
\hline caçavots 'cazavotos', adj & 0 & - & 24 & 100 & 0 & - \\
\hline catalanofóbia 'catalanofobia', f & 14 & 58,3 & 7 & 29,2 & 3 & 12,5 \\
\hline claqueter claquetera 'claquetero claquetera', $\mathrm{m}$ y $\mathrm{f}$ & 22 & 88 & 2 & 8 & 1 & 4 \\
\hline cleptòcrata 'cleptócrata', m y f & 0 & - & 25 & 100 & 0 & - \\
\hline cretinitzador $-a$ 'cretinizador -ra', adj & 0 & - & 25 & 100 & 0 & - \\
\hline méteo 'méteo', f & 1 & 4 & 24 & 96 & 0 & - \\
\hline onze de garanties 'once de garantias', $\mathrm{m}$ & 2 & 8,3 & 22 & 91,7 & 0 & - \\
\hline psendoecologista 'pseudoecologista', adj & 1 & 4 & 24 & 96 & 0 & - \\
\hline rastaflauta 'perroflauta', m y f & 2 & 8 & 23 & 92 & 0 & - \\
\hline saxofônic -a 'saxofónico -ca', adj & 14 & 56 & 5 & 20 & 6 & 24 \\
\hline seminacionalitzar 'seminacionalizar', $\mathrm{v}$ tr & 25 & 100 & 0 & - & 0 & - \\
\hline sou base 'sueldo base', $\mathrm{m}$ & 24 & 100 & 0 & - & 0 & - \\
\hline webisodi 'webisodio', $\mathrm{m}$ & 23 & 92 & 2 & 8 & 0 & - \\
\hline
\end{tabular}

En general, vemos que en el caso de los neologismos en que la coincidencia de resultados se reduce, aumenta el porcentaje de unidades clasificadas con ambos valores, es decir, con la tercera categoría. Este es el caso de saxofonic -a $(24 \%)$, catalanofobia (12,5\%), autoretallada $(8,7 \%)$ y, en menor grado, claqueter claquetera (4\%). En estos cuatro casos prima la categoría 'denominativo'. En cambio, en el caso de los neologismos en que prima la categoría 'estilístico', no observamos ningún ejemplo en que se clasifique el neologismo como 'con ambos valores'. Este hecho se explica, en parte, porque el grado de coincidencia de los resultados es mayor (igual o superior al $91,7 \%$ ). En este sentido, los casos observados anteriormente se han clasificado como denominativos con un máximo de un $88 \%$ de coincidencia que baja hasta el $56 \%$ en el menor de los casos. Como hay dos categorías principales, tienen un $50 \%$ de posibilidades de clasificación, que disminuye a un 33\% si tenemos en cuenta la tercera opción, con ambos valores, que casi no han utilizado.

En la Tabla 7 indicamos el porcentaje de resultados coincidentes a la hora de clasificar los neologismos en 'denominativos' y 'estilísticos'.

Tabla 7. Coincidencia de los resultados por categoría.

\begin{tabular}{|l|c|l|c|}
\hline Neologismos denominativos & Coincidencia (\%) & Neologismos estilísticos & Coincidencia (\%) \\
\hline autoretallada & 65,2 & caçavots & 100 \\
\hline catalanofobia & 58,3 & cleptòcrata & 100 \\
\hline claqueter claquetera & 88 & cretinitzador -a & 100 \\
\hline saxofonic -a & 56 & méteo & 96 \\
\hline seminacionalitzar & 100 & onze de garanties & 91,7 \\
\hline sou base & 100 & pseudoecologista & 96 \\
\hline webisodi & 92 & rastaflauta & 92 \\
\hline promedio & 79,9 & promedio & 96,5 \\
\hline
\end{tabular}


Como se observa, en la mayoría de los casos no coinciden las respuestas de todos los participantes, aunque en todos los neologismos los niveles de coincidencia se sitúan por encima del 50\%, sin excepciones. En este sentido, hemos clasificado los neologismos en la categoría en que hay mayor coincidencia. Por este motivo, como en las unidades con ambos valores el grado de coincidencia no llega al $50 \%$ en ninguno de los casos, se ha omitido esta tercera categoría en la Tabla 7. Así pues, vemos que 7 unidades se han considerado neologismos denominativos mientras que las otros 7 se han clasificado como neologismos estilísticos. Por otro lado, observamos que los ejemplos con un porcentaje de coincidencia menor se concentran sobre todo en relación con los neologismos clasificados como denominativos. En este sentido, el promedio de coincidencia entre los neologismos es de un $88,2 \%$ : en los neologismos considerados denominativos se sitúa en un 79,9\% mientras que en relación con los clasificados como estilísticos esta cifra aumenta hasta el 96,5\%.

De acuerdo con estos resultados, parece que, a diferencia de lo observado en la bibliografía sobre neologismos denominativos y estilísticos, en que se indica que los neologismos estilísticos son más difíciles de definir (García Platero, 1996; Faura, 1998), el resultado es a la inversa. Los datos obtenidos parecen indicar que el valor estilístico es más fácil de identificar y, por este motivo, el grado de coincidencia entre los participantes es más alto. En cambio, en el caso de los neologismos prototípicos del valor denominativo los elementos 'visibles' o 'identificables' para su clasificación como denominativos no sobresalen o no destacan, de manera que se clasifican diferentemente de acuerdo con la percepción del participante involucrado en el proceso.

En la Tabla 8 podemos ver como se reparten los neologismos de acuerdo con los datos de coincidencia obtenidos:

Tabla 8. Clasificación de acuerdo con el grado de coincidencia.

\begin{tabular}{|c|c|c|}
\hline Coincidencia (\%) & $\mathbf{N .}^{\mathbf{}}$ neologismos & Ejemplos \\
\hline 100 & $5(35,7 \%)$ & cretinitzador, seminacionalitzar \\
\hline $99-90$ & $5(35,7 \%)$ & pseudoecologista, sou base \\
\hline $89-80$ & $1(7,1 \%)$ & claqueter \\
\hline $79-70$ & $0(0 \%)$ & - \\
\hline $69-60$ & $1(7,1 \%)$ & autoretallada \\
\hline $59-50$ & $2(14,3 \%)$ & catalanofobia, saxofonic \\
\hline total & 14 & \\
\hline
\end{tabular}

El grupo de neologismos más numeroso es el formado por neologismos con un $100 \%$ de coincidencia y los que presentan un grado de coincidencia muy alto (entre un $99 \%$ y un $90 \%$ ), ya que cada uno representa algo más de una tercera parte. Los casos con una coincidencia inferior al $75 \%$ representan casi una cuarta parte. 


\subsection{Contraste con los parámetros de análisis}

\subsubsection{Frecuencia y estabilidad en el uso}

Para obtener más información sociolingüística que nos ayude a analizar con más detalle estas unidades, es decir, para evaluar y comprender los resultados de la encuesta, valoramos el uso que presentan estas unidades en el banco de datos del Observatori de Neologia. En este sentido, tenemos en cuenta tanto la frecuencia (número de ocurrencias) como la estabilidad (la primera documentación y el número de años en que se ha documentado), que son dos aspectos diferentes y complementarios.

Tabla 9. Resultados en relación con la frecuencia y la estabilidad en el uso.

\begin{tabular}{|c|c|c|c|}
\hline Neologismos & $1^{\mathrm{a}}$ doc. & Años & Ocurrencias \\
\hline \multicolumn{4}{|l|}{ Neologismos denominativos } \\
\hline webisodi 'webisodio' & 2010 & 3 & 4 \\
\hline catalanofobia 'catalanofobia' & 1993 & 2 & 2 \\
\hline sou base 'sueldo base' & 2007 & 2 & 2 \\
\hline autoretallada 'autorecorte' & 2010 & 2 & 2 \\
\hline seminacionalitzar 'seminacionalizar' & 2010 & 1 & 1 \\
\hline claqueter claquetera 'claquetero claquetera' & 2011 & 1 & 1 \\
\hline saxofonic-a'saxofónico -ca' & 2011 & 1 & 1 \\
\hline \multicolumn{4}{|l|}{ Neologismos estilísticos } \\
\hline méteo 'méteo' & 2005 & 3 & 4 \\
\hline rastaflauta 'perroflauta' & 2011 & 2 & 4 \\
\hline cleptòcrata 'cleptócrata' & 1997 & 3 & 3 \\
\hline pseudoecologista 'pseudoecologista' & 2006 & 2 & 2 \\
\hline caçavots 'cazavotos' & 2010 & 1 & 1 \\
\hline cretinitzador - $a$ 'cretinizador -ra' & 2010 & 1 & 1 \\
\hline onze de garanties 'once de garantias' & 2012 & 1 & 1 \\
\hline
\end{tabular}

En general, observamos que los neologismos de la encuesta no presentan una frecuencia de uso alta, de acuerdo con los datos del BOBNEO, porque el número de ocurrencias se encuentra en todos los casos entre 1 y 4. Concretamente, 6 de los neologismos son 'hápaxs', puesto que se documentan una sola vez en el BOBNEO, como es el caso de cacavots, cretinitzador y onze de garanties, considerados estilísticos, y claqueter claquetera, saxofonic -a y seminacionalitzar, clasificados como denominativos. Así pues, aunque Guilbert (1975) y Cabré (1989) indican que los neologismos denominativos acostumbran a tener una frecuencia de uso elevada y a estabilizar-se, vemos que no siempre es así. $\mathrm{O}$, que al menos, los hablantes perciben como denominativas unidades que no son frecuentes y estables. En el otro extremo, con 4 ocurrencias, tenemos 3 unidades: méteo y rastaflauta, clasificados como estilísticos, y webisodi, como denominativo. En este caso, vemos que, en cambio, unidades más frecuentes son consideradas estilísticas, obviando, pues, el principio de no-frecuencia.

En todos estos casos, la mayoría de los neologismos se registró por primera vez entre 2010 y 2012. Aun así, vemos 2 ejemplos ya documentados en los años noventa: 
catalanofobia, en 1993, y cleptòcrata, en 1997. Aunque en ambos casos su uso se remonta dos décadas atrás, los resultados de clasificación de acuerdo su función comunicativa no converge, puesto que catalanofóbia se ha clasificado como 'neologismo denominativo' (con el 58,3\% de coincidencia) mientras que cleptòcrata se ha considerado un 'neologismo estilístico' (con el 100\% de los resultados). Así pues, en estos casos podemos afirmar que los datos sociolingüísticos, aunque se mencionan en la bibliografía para diferenciar los dos tipos de funciones, no son suficientes para explicar las diferencias en la percepción de los participantes.

\subsubsection{Documentación en otras obras}

Para ampliar el estudio del uso de estos neologismos, observamos si estas unidades se documentan en otras obras terminológicas y enciclopédicas, y también en corpus textuales y lexicográficos. Esto indicaría que, aunque estas unidades no se documentan en los diccionarios de referencia en catalán, se utilizan de forma más o menos frecuente y, por este motivo, se documentan en otras obras lexicográficas o en corpus. En relación con el primer grupo de fuentes, los neologismos se buscaron en el Cercaterm del Termcat, un banco de datos especializado, y en la Viquipèdia, una obra enciclopédica realizada de forma voluntaria y colaborativa. Si se documentan en estas dos obras, nos indica que los neologismos hacen referencia al conocimiento especializado y enciclopédico. En el segundo caso, los neologismos se buscaron en Google Académico, un corpus más restringido que Google. Finalmente, también se observó si se habían documentado en obras no actualizadas como el Corpus textual $i$ informatitzat de la llengua catalana (CTILC) y el Diccionari català-valencià-balear (DCVB) de Alcover y Molln (2002) ${ }^{4}$. Estas obras acreditan la frecuencia y estabilidad en el uso.

Tabla 1o. Resultados de la documentación en otras obras.

\begin{tabular}{|ll|lll|}
\hline Cercaterm & Viquipèdia & \multicolumn{1}{c|}{ G. Acadèmic } & CTILC & DCVB \\
\hline \multicolumn{7}{|c|}{ Neologismos denominativos } \\
- & catalanofóbia & $\begin{array}{l}\text { catalanofobia } \\
\text { sou base }\end{array}$ & $\begin{array}{l}\text { catalanofobia } \\
\text { sou base }\end{array}$ & catalanofóbia \\
\hline \multicolumn{7}{|c|}{ Neologismos estilísticos } \\
\hline- & cleptòcrata & - & - & - \\
- & - & pseudoecologista & - & - \\
- & - & - & cretinitzador $-a$ & - \\
\hline
\end{tabular}

De acuerdo con los resultados obtenidos, catalanofobia es la unidad que disfruta de un uso más amplio y estable, puesto que se registra en tres obras: la Viquipèdia, el CTILC y el DCVB. El hecho que se documente en la Viquipèdia nos indica que catalanofobia hace referencia a un concepto relevante de nuestra sociedad, aunque no se registre en los diccionarios en catalán. De todos modos, catalanofóbia ha sido una de las unidades con un menor grado de coincidencia entre los hablantes en su clasificación en 'denominativo' (58,3\%), 'estilístico' (29,2\%) o con ambos valores (12,5\%). Por un lado, se trata de una unidad conocida, puesto que presenta un uso más o menos 
estable y frecuente. Ahora bien, desde un punto de vista lingüístico, aunque se trata de una unidad bien formada, presenta una connotación negativa, como veremos más adelante.

En relación con el resto de las unidades documentadas, diríamos que la otra unidad clasificada como denominativa, sou base, parece tener un uso más o menos estable, puesto que se documenta en dos fuentes diferentes. El resto de las unidades, todas clasificadas como estilísticas (cleptòcrata, psendoecologista y cretinitzador -a), solamente se han documentado en una obra, hecho que indica que su uso no parece haberse estabilizado. De todos modos, cabe destacar que cleptòcrata se documenta en la Viquipèdia, ya que indicaría que se trata una unidad que hace referencia a un conocimiento compartido del mundo y, por este motivo, se ha incluido en esta obra enciclopédica.

Para terminar, podemos añadir que el hecho que solamente 6 de las 14 unidades se documenten en las obras seleccionadas, no permite extrapolar los resultados obtenidos. Aunque es esperable que no se documenten, porque se trata de unidades recientes, el hecho que se documenten tanto unidades consideradas denominativas como estilísticas permite observar que la frecuencia de uso y la estabilidad no es un argumento suficiente a la hora de caracterizar los neologismos de acuerdo con su función comunicativa. Como indica Llopart-Saumell (2016), debe ponerse en relación con los demás criterios.

\subsubsection{Posición discursiva y marcas de uso en contexto}

Por lo que respecta a la posición discursiva, debemos tener en cuenta que el discurso periodístico se divide en dos macroestructuras, la narrativa periodística o relato, y la opinión periodística o comentario, que hay que diferenciar para separar información y opinión (Martínez Albertos, 1989; Casals Carro, 2011). De acuerdo con estos autores, en el primer caso se explican o se transfieren unos hechos, en el que se incluyen las noticias, las crónicas y los reportajes. En el segundo caso, en cambio, se opina sobre el valor de los hechos, es decir, se expresan ideas, juicios y pensamientos, como se observa en los artículos de opinión (editoriales, columnas, tribunas, ensayos, críticas y declaraciones). Por este motivo, tenemos en cuenta si el neologismo se documenta en una sección de opinión, porque contiene elementos subjetivos. En cuanto a la posición discursiva del neologismo, también valoramos si el neologismo se documenta en el título de la noticia o en el cuerpo del texto. En este sentido Calsamiglia y Tusón (2012) explican que los titulares se consideran enunciados con una función catafórica, porque se usan como señuelo para captar la atención de los posibles lectores. De este modo, esperaríamos que estuviera más relacionado con los neologismos estilísticos.

En cuanto al contexto discursivo, a continuación, presentamos algunos aspectos gramaticales no prototípicos de los textos de prensa de acuerdo con varios manuales 
de estilo (Martínez Albertos, 1989; Grijelmo, 1997/2001; El País, 2014), pero observables en el contexto de uso en que se encuentra el neologismo. Por ejemplo, elementos propios de la lengua coloquial y también de la lengua pseudoliteraria. La lengua coloquial está estrechamente relacionada con la informalidad, la expresividad y la subjetividad. Y, por otra parte, la relación que mantiene esta modalidad lingüística con el componente expresivo también se observa en otro tipo de lengua, la literaria. Así pues, estas dos modalidades comparten algunos rasgos propios de la búsqueda de expresividad y con un objetivo estético. De acuerdo con esta información, pues, también nos proponemos identificar en el contexto del neologismo los elementos característicos de la lengua literaria, como son las figuras retóricas o el adjetivo ornamental (antepuesto al nombre). Por otro lado, también tenemos en cuenta si hay deixis de emisor, ya que de acuerdo con Calsamiglia y Tusón (2012), la figura del 'yo' está relacionada con la subjetividad en el lenguaje y no es esperable (ni deseable) en textos objetivos. Finalmente, observamos si presentan marcas tipográficas (negrita, cursiva, comillas) o metadiscursivas (sinonimia, perífrasis, equivalentes, apoyo gramatical, etc.).

En este sentido, entendemos por coloquialismo, una palabra o expresión marcada en los diccionarios como coloquial, popular o vulgar. Con esta etiqueta también incluimos las palabras propias del argot, insultos, eufemismos, frases hechas y refranes, aumentativos y diminutivos, independientemente de si se documentan en los diccionarios consultados. En cuanto a las figuras retóricas, tenemos en cuenta las metáforas (no documentadas en los diccionarios), la metonimia, la hipérbole (marcada o no en los diccionarios), la antítesis y las preguntas retóricas, entre otras.

En la Tabla 11 se indica si se encuentran o no estos elementos en el contexto del neologismo y, si es así, se especifica de que tipo son.

Tabla 11. Resultados de acuerdo con los aspectos gramaticales, las marcas de uso del contexto y la posición discursiva.

\begin{tabular}{|l|l|l|l|}
\hline Neologismos & Posición discursiva & Aspectos gramaticales & Marcas \\
\hline \multicolumn{2}{|c|}{ Neologismos denominativos } \\
\hline autoretallada & opinión & - & - \\
\hline webisodi & - & - & cursiva \\
\hline \multicolumn{2}{|c|}{ Neologismos estilísticos } \\
\hline rastaflauta & título, opinión & emisor & sinónimo \\
\hline cretinitzador -a & título, opinión & $\begin{array}{l}\text { metáfora, pregunta } \\
\text { retórica }\end{array}$ & - \\
\hline cleptòcrata & declaraciones & adjetivo & - \\
\hline pseudoecologista & - & $\begin{array}{l}\text { coloquialismo, } \\
\text { emisor }\end{array}$ & - \\
\hline méteo & - & - & cursiva \\
\hline
\end{tabular}

Como vemos, la mitad de los neologismos (7 unidades) presentan las marcas que hemos indicado. Se trata de autoretallada, cleptòcrata, cretinitzador -a, méteo, pseudoecologista, 
rastaflauta y webisodi. La mitad restante, en cambio, no presentan estos rasgos en el contexto de uso. De estas 7 unidades, 5 fueron consideradas estilísticas por la mayoría de los participantes (con una coincidencia superior o igual al 92\%) mientras que únicamente autoretallada y webisodi fueron consideradas denominativas (con el 60\% y el $92 \%$ de coincidencia, respectivamente). Así pues, a partir de los datos obtenidos parece observarse una correlación entre la intuición lingüística de los hablantes para considerar como denominativos o estilísticos estos neologismos y las marcas de uso observables en el contexto o que se desprenden de su posición discursiva.

En relación con los aspectos propios de la lengua coloquial y pseudoliteraria, podemos observar algunos ejemplos como los siguientes:

(1) Contemplant, amb estupor, la deriva cretinitzadora de la nostra cultura: es possible un retorn crític $i$ meditat a un món en què una teoria científica o una pel·lícula de qualitat no s'ubicaven en el mateix prestatge dels prejudicis irracionals o dels programes televisius d'assumptes del cor? [Avin, 23/02/2010]

(2) Res més lluny de la meva intenció que apuntar-me a un cavall guanyador -a més, Tinto Brass no és ni una cosa ni l'altra-, però he de confessar que jo també vaig tenir una revelació semblant quan em vaig empassar estoicament la faula tonta i pseudoecologista de James Cameron. [El Periódico, 2/03/2010]

(3) Per tant no s'ofendran els okupants si ens hi referim com a rastaflautes. [ $\mathrm{La}$ Vanguardia, 23/06/2011]

(4) El president de la Generalitat del País Valencià s’ha passat de la ratlla quan ha volgut condicionar el rescat econòmic a una autoretallada a les institucions d'autogovern. [Ara, 28/07/2012]

En relación con la lengua coloquial, observamos locuciones y expresiones propias de este registro como 'tonto', 'apuntarse a caballo ganador', 'pasarse de la raya' o 'no era ni una cosa ni la otra'. También detectamos el uso de algunas figuras retóricas como metáforas o preguntas retóricas, observable en el contexto de cretinitzador - $a$. Por ejemplo, 'ubicarse en la estantería de los prejuicios irracionales'. Además, vemos que se ha redactado en primera persona del singular o del plural, y se utilizan verbos de sentimiento ('nos referimos', 'debo confesar', etc.). Estas figuras aportan un toque literario al texto además de emotividad, crítica y deseo.

En lo que respecta a méteo y webisodi, ambos casos se han marcado con cursiva. Cabe señalar que las marcas tipográficas se utilizan con finalidades diversas, por ejemplo, para introducir un énfasis especial, destacar la unidad, indicar que es una unidad argótica, etc. Finalmente, en el contexto de rastaflauta, este neologismo se 
utiliza como un hipónimo de okupante, ya que se indica "no se ofenderán los okupantes si nos referimos a ellos como perroflautas".

(5) Consulti la méteo en l'últim cap de setmana d'agost. [La Vanguardia, 27/08/2011]

(6) I fins i tot ha penjat a Youtube diversos webisodis en què el mateix artista del Prat narra els seus orígens, tenint com a punt de partida el col-legi on va estudiar i la vorera on va veure uns nois tocant flamenc que van fer que s'enamorés de la música. [El Periódico, 23/07/2012]

Para terminar, en relación con la posición y el género discursivo vemos que autoretallada, cleptòcrata, cretinitzador - a y rastaflauta se han documentado en artículos de opinión o en declaraciones. Además, tanto cretinizador como rastaflauta aparecen en el título del artículo para llamar la atención del lector. Como indica Sablayrolles (2000), algunos de los neologismos de prensa se sitúan sobre todo en el titulo para llamar la atención, porque en el cuerpo de la noticia tienen una función de focalización.

\subsection{Tipo de formación, y aspectos morfosemánticos y pragmáticos}

En relación con el proceso de formación que han seguido para crearse, cabe destacar tanto el mecanismo como también otras cuestiones morfológicas, semánticas y pragmáticas. En relación con los tipos de formación de los neologismos seleccionados para este experimento, las unidades se incluyeron de manera que se observa una representación equilibrada de los distintos mecanismos. En este sentido, cabe destacar que se han tenido en cuenta únicamente las formas nuevas. Aunque en este caso no podemos analizar con profundidad el impacto del proceso de formación, en algunos estudios se ha puesto de manifiesto el peso de esta variable para identificar los neologismos (Sablayrolles, 2003) y también en relación con la función comunicativa que llevan a cabo. Concretamente, en Llopart-Saumell (2017) se presentan datos empíricos que confirman la correlación entre la función de los neologismos y los procesos de formación, como habían postulado Guilbert (1975) y Cabré (1989). Por otro lado, si observamos con más detalle cómo se han formado las distintas unidades, podemos explicitar algunas cuestiones en relación con la predictibilidad de las formas resultantes, en relación con la lexicalización de algunas unidades poliléxicas y el valor pragmático que desprenden.

En cuanto a cretinitzador -a, por ejemplo, se ha formado a partir de la base cretí -ina 'cretino -na', que es una unidad coloquial y peyorativa que significa 'estúpido', y el sufijo en catalán -dor del latín -tor, -toris, que tiene dos acepciones. En la primera, adopta el significado de 'gente o profesión' como en 'tejedor'. La segunda acepción indica 'relación con verbos' como se observa en 'conocedor'. Ahora bien, por lo que respecta a 'cretinitzador' $-a$, sabemos que no hace referencia a una persona, porque en 
el contexto actúa como adjetivo (deriva 'cretinitzadora'). Además, el verbo 'cretinitzar' no existe en catalán (tampoco en castellano) y, por tanto, también se consideraría una unidad neológica. El neologismo 'pseudoecologista' también llama la atención porque contiene una forma prefijada con una connotación negativa, pseudo-. Finalmente, podemos añadir que estas connotaciones también presentan un carácter subjetivo y afectivo.

Por otro lado, el neologismo 'rastaflauta' es un compuesto coordinado $[\mathrm{N}+\mathrm{N}]_{\mathrm{N}}$ en que el componente 'rasta' es un neologismo formado por abreviación de 'rastafari' (no documentado en los diccionarios de referencia en catalán), que proviene del nombre propio del amárico Ras Tafari (Oxford English Dictionary). Ahora bien, como se observa en el contexto, esta palabra se utiliza de forma despectiva para hablar de una persona joven con un aspecto desaliñado. En cuanto a la formación, semánticamente no es predictible en el sentido que las dos unidades que forman el compuesto no pertenecen al mismo campo conceptual como sucede en general en los compuestos coordinados, especialmente en nombres de profesiones, como en 'magistrado juez' (Bernal, 2012). En Cabré, Freixa y Solé (1997/2002) también hablan de incompatibilidad cuando los componentes coordinados presentan características diferentes.

En cuanto a catalanofobia, está formado por las formas cultas 'catalano'-, de catalán, y -fóbia ('-fobia'). Si observamos los ejemplos del DIEC2, esta forma se combina con otras formas cultas para hacer referencia a diferentes patologías (hidrofobia, fotofobia, etc.) aunque más recientemente también se utiliza en el sentido de 'aversión' o 'rechazo'.

(7) En plena disputa pel nou model de finançament el març del 2008, Aguirre sorprenia espolsant-se el retret de promoure la catalanofòbia anunciant durant un esmorzar amb militants a Lleida la creació imminent de la primera escola bilingüe castellà-català a la capital de l'Estat. [Avii, 17/11/2010]

Algunos neologismos expresan precisión léxica, de acuerdo con Mounin (1990) y Sablyarolles (1993), o especifican el concepto al cual hacen referencia. Por ejemplo, el compuesto caçavots, que presenta una estructura $[\mathrm{V}+\mathrm{N}] \mathrm{N}$ y funciona como adjetivo, y se utiliza para especificar la característica del nombre al cual acompaña inversions caçavots'. En este sentido, aunque sigue formas usuales, se crean para la expresión del momento y no se difunde en el uso. En este caso, se indica la intencionalidad con que se realiza esta inversión. Lo mismo ocurre con el adjetivo saxofonic - $a$, en que se especifica el tipo de relación semántica. Este neologismo se ha formado a partir de la adjunción de sufijo - $i$-ica, que significa 'relativo o perteneciente a', a la base saxofon. Así pues, aunque se trata de una unidad posible de acuerdo con las reglas de formación, presenta una frecuencia de uso baja. En ese sentido, este adjetivo sustituye la perífrasis de saxofón o con el saxofón. 
(8) Sorpreses saxofòniques amb Esther Sabaté i Jordi Farran. [El Periódico, 6/06/2011]

Otros, como claqueter claquetera, expresan economía lingüística. Se trata de una forma predictible que hace referencia a la persona que practica este baile, y que se ha formado adjuntando el sufijo -er -era, que significa 'profesional de', a la base claqué. De esta manera, se evita el uso de la perífrasis ballarí de claqué.

(9) A més dels claqueters, també ballarins de lindy hop o amants del swing es trobaran per ballar el Shim sham, un pas popular que interpreten tant principiants com professionals del claqué i també de la família del swing. [Avii, 23/05/2011]

En relación con el neologismo sintagmático del ámbito de los deportes con sentido metafórico, se trata de onze de garanties. Como podemos ver, presenta un significado compositivo, y hace referencia al equipo titular en un partido de fútbol compuesto por 11 jugadores de un equipo.

(10) Actualment, amb els dotze futbolistes provinents de les categories inferiors que formen la plantilla, faltarien jugadors que ocupessin llocs defensius per completar un onze de garanties, ja que tan sols Puyol, Piqué i el lesionat Fontàs són defenses purs. [Ara, 18/02/2012]

El neologismo seminacionalitzar presenta la categoría gramatical verbo, que no es tan común como los nombres a la hora de crear unidades nuevas de la lengua. En más detalles, al vebro nacionalitz̧ar se ha añadido el prefijo semi-, que indica una gradación de la intensificación en el sentido de 'parcialmente, no completamente, casi'.

(11) El dia va començar amb la notícia que el seminacionalitzat Commerzbak va presentar uns resultats horribles. [El Periódico, 24/02/2010]

Por lo que respecta a sou base, se trata de un compuesto $[\mathrm{N}+\mathrm{Adj}]_{\mathrm{N}}$ que es un sinónimo de salari base.

(12) Els que superen els 3.000 euros bruts com a sou base són l'excepció. [El Periodico, 06/06/2011]

Finalmente, el neologismo webisodi es un acrónimo formado a partir del préstamo del inglés web (ya documentado en las principales obras de referencia en catalán, y también del castellano) y episodi. Así pues, aunque se trata de una formación por acronimia podemos indicar que se trata de una unidad híbrida. Se trata de un tipo de episodio o serie. En cuanto a méteo, se trata de una abreviación de 'meteorologia'.

De acuerdo con estas observaciones, podemos afirmar que los neologismos que se desvían de la norma o de las reglas de formación acostumbran a tener una motivación que va más allá de la meramente referencial. Por esta razón, acostumbran a percibirse, 
también, como estilísticas, porque son sorprendentes. También ocurre lo mismo con las unidades que presentan connotaciones negativas, como es el caso de catalanofobia. Sin embargo, al contrario que las demás unidades, su uso es más frecuente y estable. En los neologismos en que se observa una metáfora en el sí de la unidad, como en onze de garanties, si esta metáfora se ha lexicalizado, la sorpresa que causa en el receptor disminuye. Pero, como en este caso, si no es así, se percibe como una unidad connotada y con valor pragmático. En relación con las formas híbridas, como webisodi, aunque no son esperables de acuerdo con las reglas de formación, se trata de formas bastante productivas $y$, por tanto, frecuentes. Por este motivo, también dejan de percibirse como sorprendentes.

\section{CONCLUSIONES}

En relación con los resultados de la encuesta, el promedio de coincidencia se sitúa en el 88,2\%. En un 70,1\% de los neologismos (10 de las 14 unidades) el grado de coincidencia se sitúa entre el 90 y el 100\%. Por otro lado, la mitad (35,7\%), es decir, 5 unidades, presentan un pleno de resultados. El dato más destacado es el alto grado de coincidencia en la clasificación de los neologismos considerados estilísticos, puesto que el promedio de las 7 unidades clasificadas en esta categoría es del 96,5\%. Además, en todos los casos la coincidencia de los resultados se sitúa por encima del $90 \%$ y ninguno de los neologismos ha sido considerado con ambos valores, es decir, tanto denominativo como estilístico. En cambio, si observamos los datos relativos a los neologismos clasificados mayormente como denominativos, no observamos el mismo grado de coincidencia. En este caso, solamente 3 de las 7 unidades presentan un grado de coincidencia superior al 90\% y 2 de ellas se sitúan por debajo del 60\% (catalanofobia y saxofonic -a), que han sido consideradas estilísticas por algunos participantes y también con ambos valores, por otros.

Finalmente, en relación con la correlación de los datos de la encuesta con el estudio de aspectos sociolingüísticos, discursivos y lingüísticos, relacionados en la bibliografía sobre la dicotomía centrada en la función de los neologismos de acuerdo con las categorías 'denominativa' y 'estilística', hemos observado que, por separado, estos factores no son suficientes para relacionarlos con la función comunicativa. Sin embargo, se complementan. En definitiva, el que ha resultado más indicativo ha sido el aspecto lingüístico. Concretamente, el estudio de los aspectos morfosemánticos respecto de la norma y las reglas de formación de palabras. 


\section{REFERENCIAS BIBLIOGRÁFICAS}

Alcover, A. M. \& Moll, F. de B. (2002). Diccionari català-valencià-balear [DCVB] [en línea]. Disponible en: http://dcvb.iecat.net/

Bernal, E. (2012). Catalan compounds. Probus, 24(1), 5-27.

Bernal, E. (2015). Ser o no ser: Els neologismes i la percepció dels parlants, entre la normalitat i la raresa. En C. Sinner \& K. Wieland (Eds.), Norma, ús $i$ actituds lingüistiques: el paper del català en la vida quotidiana (pp. 61-73). Leipzig: Leipziger Universitätsverlag.

[BOBNEO] Observatori de Neologia. (2016). Cercador BOBNEO [en línea]. Disponible en: http://obneo.iula.upf.edu/bobneo/index.php

Brown, G. \& Yule, G. (1991). Discourse analysis. Cambridge: Cambridge University Press.

Cabré, M. T. (1989). La neologia efímera. En J. Massot (Coord.), Miscel-lània Joan Bastardas, 1 (Estudis de Llengua $i$ Literatura Catalanes, XVIII) (pp. 37-58). Barcelona: Publicacions de l'Abadia de Montserrat.

Cabré, M. T., Freixa, J. \& Solé, E. (1997/2002). À la limite des mots construits possibles. En M. T. Cabré, J. Freixa \& E. Solé (Eds.), Lèxic i neologia (pp. 121138). Barcelona: Observatori de Neologia, Institut Universitari de Lingüística Aplicada, Universitat Pompeu Fabra.

Calsamiglia, H. \& Tusón, A. (2012). Las cosas del decir: Manual de análisis del discurso. Barcelona: Ariel.

Cañete, P. (2015). Innovación léxica y género en textos periodísticos del español actual. Tesis doctoral, Universitat Pompeu Fabra, Barcelona, España [en línea]. Disponible en: http://www.tesisenred.net/handle/10803/392898

Casals Carro, M. J. (2011). El universo retórico del periodismo. En M. L. Sánchez Calero (Ed.), Géneros y discurso periodístico (pp. 45-73). Madrid: Fragua.

Casasús, J. M. (1992). El lenguaje periodístico ante el neologismo. En Agencia Efe (Ed.), El neologismo necesario (pp. 87-90). Madrid: Fundación Efe.

[Cercaterm] Termcat. (2016). Cercaterm [en línea]. Disponible en: http://www.termcat.cat/es/Cercaterm/Fitxes/

[CTILC] Institut d'Estudis Catalans. (2005). Corpus textual informatitrat de la llengua catalana [en línea]. Disponible en: http://ctilc.iec.cat/

Cortés, L. \& Camacho, M. M. (2003). ¿Qué es el análisis del discurso? Barcelona: EUB. 
[DIEC2] Institut d'Estudis Catalans (Ed.). (2007). Diccionari de la llengua catalana [en línea]. Barcelona: Edicions 62 - Enciclopèdia Catalana [en línea]. Disponible en: http://dlc.iec.cat/

El País (2014). Libro de estilo (22a ed.). Madrid: Aguilar.

Escandell-Vidal, M. V. (1996). Introducción a la pragmática. Barcelona: Ariel.

Escandell-Vidal, M. V. (2014). La comunicación: Lengua, cognición y sociedad. Madrid: Akal.

Estopà, R. (2015). Sobre neologismos y neologicidad: Reflexiones teóricas con repercusiones metodológicas. En I. M. Alves \& E. Simoes Pereira (Eds.), Neologia das Linguas Romanicas (pp. 111-150). Sao Paulo: CAPES, Humanitas.

Estornell, M. (2009). El reconocimiento de neologismos y su caracterización en un corpus de prensa escrita (2004-2007). Tesis doctoral, Universitat de València, València, España [en línea]. Disponible en: http://www.tdx.cat/handle/10803/9827

Faura, N. (1998). Futbol i llenguatge: La innovació lèxica a les cròniques $i$ a les retransmissions futbolístiques. Barcelona: Publicacions de l'Abadia de Montserrat.

Freixa, J. (2002). Dimensió social de la neologia. En M. T. Cabré, J. Freixa \& E. Solé (Eds.), Lèxic i neologia (pp. 69-78). Barcelona: Observatori de Neologia Institut Universitari de Lingüística Aplicada - Universitat Pompeu Fabra.

Freixa, J. (2010). Paraules amb rareses. Terminàlia, 1, 7-16.

García Platero, J. M. (1996). Observaciones sobre el neologismo. Revista de Lexicografia, 2, 49-59.

Gardin, B., Lefèvre, B., Marcellesi, C. \& Mortureux, M.-F. (1974). À propos du «sentiment néologique». Langages, 8(36), 45-52.

[GDLC] Enciclopèdia Catalana. (1998). Gran diccionari de la llengua catalana [en línea]. Disponible en: http://www.diccionari.cat/

Google. (2016). Google Acadèmic [en línea]. Disponible en: https://scholar.google.es

Grijelmo, Á. (1997/2001). El estilo del periodista (7a ed.). Madrid: Taurus.

Guerrero Ramos, G. (1995). Neologismos en el español actual. Madrid: Arco Libros.

Guilbert, L. (1975). La créativité lexicale. París: Larousse. 
Isla, J. D. (2006). El español en la prensa. Neología y registros lingüísticos: Los neologismos en suplementos juveniles de diarios nacionales. Actas de las IX Jornadas Nacionales sobre Normativa del Idioma Español. Buenos Aires: Fundación Litterae [en línea]. Disponible en: http://www.fundlitterae.org.ar/images/archivos/ISLA.doc

Llopart-Saumell, E. (2016). La funció dels neologismes: Revisió de la dicotomia neologisme denominatiu $i$ neologisme estilístic. Tesis doctoral, Universitat Pompeu Fabra, Barcelona, España [en línea]. Disponible en: http:/ / tesisenred.net/handle/10803/398142

Llopart-Saumell, E. (2017). La (inter)relació entre la funció comunicativa dels neologismes i el procés de formació. Terminàlia, 16, 7-17 [en línea]. Disponible en:

http://revistes.iec.cat/index.php/Terminalia/article/view/142658/pdf_1315

Llopart-Saumell, E., Loureda, Ó., Cabré, M. T. \& Freixa, J. (2014). An experimental approach to the study of neologisms. Ponencia presentada en el Open Lab Day 2014! - Psycholinguistik zum Anfassen, Universität Heidelberg, Heidelberg.

Martínez Albertos, J. L. (1989). El lenguaje periodístico. Madrid: Paraninfo.

Mejri, S. (1995). La néologie lexicale. Tunis: Publications de la Faculté des Lettres de la Manouba.

Mortureux, M.-F. (1984). La dénomination: approche socio-linguistique. Langages, 76, 95-112.

Mounin, G. (1990). Quelques observations sur le lexique français d'aujourd'hui. Europe, 738, 10-18.

Oxford University Press. (2015). Oxford English Dictionary [en línea]. Disponible en: http://www.oed.com/

Sablayrolles, J.-F. (1993). Fonctions des néologismes. Les Cahiers du CIEL, 7, 53-94.

Sablayrolles, J.-F. (2000). La néologie en français contemporain: Examen du concept et analyse de productions néologiques récentes. Paris: Honoré Champion.

Sablayrolles, J.-F. (2003). L’innovation lexicale. Paris: Honoré Champion.

Sánchez Manzanares, M. C. (2013). Valor neológico y criterios lexicográficos para la sanción y censura de neologismos en el diccionario general. Sintagma, 25, 111125.

Van Dijk, T. A. (1989). Estructuras y funciones del discurso: Una introducción interdisciplinaria a la lingüistica del texto y a los estudios del discurso. México, D.F.: Siglo XXI. 
Varo, C. (2013). Aproximación teórico-práctica al procesamiento lingüístico de neologismos léxicos. Revista Signos. Estudios de Lingüística, 46(81), 11-12.

Veny, J. (1991). Mots d'ahir i mots d'avui. Barcelona: Empúries.

Viquipèdia. (2015). Viquipèdia, l'enciclopèdia lliure [en línea]. Disponible en: https://ca.wikipedia.org/

\section{NOTAS}

${ }^{1}$ Este estudio se ha realizado en el marco del proyecto Neómetro: La medición de la neologicidady la diccionariabilidad de los neologismos del español, financiado por el Ministerio de Economía y Competitividad (ref. FFI2016-79129-P, AEI/FEDER, UE).

2 Aunque en la encuesta participaron 25 estudiantes, la suma de la información sociolingüística da un total de 24 porque uno de los participantes no indicó los datos sociolingüísticos.

${ }_{3}^{3}$ Diccionari de la llengua catalana del Institut d'Estudis Catalans (DIEC2) y el Gran diccionari de la llengua catalana de Enciclopèdia Catalana (GDLC).

${ }^{4}$ El DCVB se editó en los años 60 y el CTILC contiene datos hasta el año 1988. 